\title{
Anomalous viscoelasticity near the isotropic-nematic phase transition in liquid crystals
}

\author{
Prasanth P. Jose ${ }^{\mathrm{a})}$ and Biman Bagchi ${ }^{\mathrm{b})}$ \\ Solid State and Structural Chemistry Unit, Indian Institute of Science, Bangalore-560012, India
}

\begin{abstract}
Recent optical Kerr effect experiments have shown that orientational relaxation of nematogens shows a pronounced slow down of the response function at intermediate times and also a power law decay near the isotropic-nematic $(I-N)$ transition. In many aspects, this behavior appears to be rather similar to the ones observed in the supercooled liquid near-glass transition [Cang et al., J. Chem. Phys. 118, 9303 (2003)]. We have performed molecular dynamics simulations of model nematogens (Gay-Berne with aspect ratio 3) to explore the viscoelasticity near the $I-N$ transition and also investigated the correlation of viscoelasticity (if any) with orientational relaxation. It is found that although the viscosity indeed undergoes a somewhat sharper than normal change near the $I-N$ transition, it is not characterized by any divergencelike behavior (like the ones observed in the supercooled liquid). The rotational friction, on the other hand, shows a much sharper rise as the $I-N$ transition is approached. Interestingly, the probability distribution of the amplitude of the three components of the stress tensor shows anisotropy near the $I-N$ transition-similar anisotropy has also been seen in the deeply supercooled liquid [Phys. Rev. Lett. 89, 25504 (2002)]. Frequency dependence of viscosity shows several unusual behaviors: (a) There is a weak, power law dependence on frequency $\left[\eta^{\prime}(\omega) \sim \omega^{-\alpha}\right]$ at low frequencies and (b) there is a rapid increase in the sharp peak observed in $\eta^{\prime}(\omega)$ in the intermediate frequency on approach to the $I-N$ transition density. These features can be explained from the stress-stress time correlation function. The angular velocity correlation function also exhibits a power law decay in time. The reason for this is discussed.
\end{abstract}

\section{INTRODUCTION}

A system of rodlike molecules with aspect ratio larger than certain minimum value is known to undergo a weak first-order phase transition from orientationally disordered to orientationally ordered (but positionally disordered) nematic state at low temperature and/or at high density. ${ }^{1,2}$ The orientational dynamics of the rodlike molecules near the isotropicnematic $(I-N)$ phase transition shows certain anomalies, the most notable being the pronounced temporal power law decay in the collective orientational correlation function discovered recently by Fayer and co-workers. ${ }^{3-8}$ A mode coupling theory analysis explains this power law decay in terms of large and rapidly growing orientational correlation near the $I-N$ transition. ${ }^{7,8}$ Theoretical analysis has quantified this growth of orientational pair correlation in an adequate manner. ${ }^{9}$ A density functional theory analysis of the $I-N$ transition has also been presented. ${ }^{10,11}$

Several computer simulations have studied orientational dynamics near the $I-N$ transition. ${ }^{12-17}$ Earlier studies have found the signature of slowdown of orientational relaxation in general. However, the simulations were not carried out for sufficiently long time to observe the decay of the relaxation function near the $I-N$ transition. Very long time molecular dynamics (MD) simulation of present authors in a system of Gay-Berne ellipsoids has observed the power

\footnotetext{
${ }^{a}$ Electronic mail: jose@sscu.iisc.ernet.in

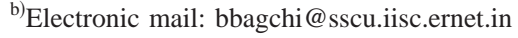

law relaxation of the collective orientational correlation function. ${ }^{17}$

An important transport property of the liquid is its shear viscosity. In the nematic phase, the shear viscosity is anisotropic. In a recent molecular dynamics simulation of the shear viscosity of Gay-Berne ellipsoids of aspect ratio 3, Smondyrev et al. calculated the temperature dependence of rotational and shear viscosities. They obtained temperature variation of Miesowicz viscosities ${ }^{18,19}$ which were defined as combinations of the components of shear viscosity but now they are defined in the nematic phase with respect to the direction of the director.

Computer simulations and theoretical analysis show that in dense, isotropic, liquid, the shear viscosity may be expressed as $^{20-23}$

$$
\eta_{\alpha \beta}(t)=\eta_{\alpha \beta}^{B}(t)+\eta_{\alpha \beta}^{\rho}(t),
$$

where $\eta_{\alpha \beta}^{B}(t)$ is the short time part that arises from static correlations which can be approximated by a Gaussian function $^{23}$ and $\eta_{\alpha \beta}^{\rho}(t)$ is the long time part that arises from the density mode contribution. An analytic expression for the $\eta_{\alpha \beta}^{\rho}(t)$ can be obtained from mode-coupling theory $(\mathrm{MCT}),{ }^{22}$

$$
\eta_{\alpha \beta}^{\rho}(t)=\frac{k_{B} T}{60 \pi^{2}} \int_{0}^{\infty} d k k^{4} \frac{\left[S^{\prime}(k)\right]^{2}}{[S(k)]^{4}} \int_{0}^{\infty} d t[F(k, t)]^{2},
$$


where $S(k)$ and $S^{\prime}(k)$ are structure factor and its first derivative, respectively, and $F(k, t)$, the intermediate scattering function, is defined by the following relation:

$$
F(k, t)=\frac{1}{N}\langle\rho(-\mathbf{k}, 0) \rho(\mathbf{k}, t)\rangle,
$$

where $\rho(\mathbf{k}, t)=\Sigma_{i} \exp \left[-i \mathbf{k} \cdot \mathbf{r}_{i}(t)\right]$. In the supercooled liquid, the decay of the dynamic structure factor slows down. This leads to an increase in the value of the shear viscosity. As discussed by Geszti, this increase in viscosity in turn further slows down the relaxation of $F(k, t)$. This feedback mechanism leads to rapid increase of viscosity in the supercooled liquid state. An important point here is that the frequency dependent viscosity is expected to closely follow the frequency dependence of the dynamic structure factor which is the Fourier transform of the intermediate scattering function.

It is known that the rotational correlation function rapidly slows down and indeed shows anomalous behavior near the $I-N$ transition. Therefore, it seems natural to ask whether the slow down in orientational relaxation has any significant effect on the variation of shear viscosity near the transition region. In this work, the shear viscosity of the a system of Gay-Berne ellipsoids is calculated along the density axis near $I-N$ transition. The shear viscosity of the system shows a rise, sharper than normal, as the $I-N$ transition is approached along the density axis. However, shear viscosity does not diverge near the $I-N$ transition. In fact, the emergence of long range orientational correlation near the $I-N$ transition is marked by the appearance of an anisotropy in the stress distribution function. Different components of the stress-stress correlation function also behave differently due to the anisotropy caused by the orientational ordering. The angular velocity autocorrelation function shows a power law decay in the long time, with an exponent -1.2 near the $I-N$ transition. This result differs from the hydrodynamic prediction of $-5 / 2$ indicating that asymptotic long time decay has not been attained in simulations. ${ }^{24-27}$

In an important work, Tang, Evans, Mason, and Allen have earlier investigated viscoelasticity of hard ellipsoids. ${ }^{28}$ Both generalized kinetic theory and computer simulations were used. These authors observed several anomalies in the frequency dependence of the real part of the frequency dependent viscosity: (i) The decrease of viscosity with frequency $\omega$ at low frequencies is very weak and can be described by a power law. (ii) The viscosity shows a pronounced minimum at intermediate frequencies not observed in atomic liquids. The authors explained the latter result as due to the coupling between the stress and the collective orientation. Tang et al. also considered the applicability of the Debye-Stokes-Einstein relation between rotational diffusion and viscosity. The viscosity approaches a constant value at high frequency, which is due to hard ellipsoid nature of the interactions.

The present simulations reveal somewhat different frequency dependence of shear viscosity near the isotropicnematic phase transition. The weak frequency dependence at low frequency exists and so does the minimum at somewhat higher frequency. The notable differences are a peak at the intermediate frequency and the approach to zero at very large frequency. The latter is of course expected for a continuous potential. The rest can be understood from an analysis of the stress time correlation function. The weak $\omega$ dependence at low $\omega$ can be traced back to a power law dependence of the stress time correlation function (STCF) while the minima and the maxima are due to damped oscillations in STCF (note the negative region in the stress TCF). The frequency dependence of shear viscosity looks similar to that of dynamic structure factor of dense liquids ${ }^{21}$ except the rapid sharpening of the maximum is clearly a signature of the translation-rotation coupling. In addition, we find that the hydrodynamic relation between the rotational friction and the viscosity breaks down completely on approach to the $I-N$ phase transition.

The organization of the rest of the paper is as follows: In the following section, we present the basic theoretical expressions required for the computation of the viscoelasticity of the system. In Sec. III, we discuss the system and the simulation details. In Sec. IV, we present the results and discuss their significance. Section V concludes with a few remarks.

\section{BASIC THEORETICAL EXPRESSIONS}

Consider a system of $N$ nematogens. The instantaneous state of the system in the phase space is specified by position $\mathbf{r}_{i}$, linear momentum $\mathbf{p}_{i}$, orientation $\boldsymbol{\Omega}_{i}$, and angular momentum $\mathbf{g}_{\boldsymbol{\Omega}_{i}}$, where $i=1, \ldots, N$. The $\alpha \beta$ component of shear viscosity of this system in the isotropic phase is given by the relation. ${ }^{18,21}$

$$
\eta_{\alpha \beta}=\frac{V}{k_{B} T} \int_{0}^{\infty} d t\left\langle\sigma_{\alpha \beta}(t) \sigma_{\alpha \beta}(0)\right\rangle,
$$

where $V$ is the volume of the system, $k_{B}$ is the Boltzmann constant, $T$ is the temperature, and $\sigma_{\alpha \beta}$ is the shear stress tensor. ${ }^{29,30}$ Shear stress tensor is defined as

$$
\sigma_{\alpha \beta}=\frac{1}{V}\left(\sum_{i} \frac{p_{\alpha i} p_{\beta j}}{m}+\sum_{i} \sum_{j>i} r_{\alpha i j} f_{\beta i j}\right),
$$

where $\mathbf{r}_{i j}$ and $\mathbf{f}_{i j}$ are relative position and force between a pair of molecules, respectively. The indices $\alpha, \beta=x, y, z$ and $\alpha \neq \beta$. The normalized stress-stress correlation function of the system is given by the expression

$$
C_{\alpha \beta}^{\sigma}(t)=\frac{\left\langle\sigma_{\alpha \beta}(t) \sigma_{\alpha \beta}(0)\right\rangle}{\left\langle\sigma_{\alpha \beta}(0) \sigma_{\alpha \beta}(0)\right\rangle} .
$$

Viscoelasticity of the system is given by frequency dependent viscosity $\eta_{\alpha \beta}(\omega)$ (Refs. 28 and 31) which is defined by the Fourier transform of $\eta_{\alpha \beta}(t)$. Viscoelasticity can be decomposed into real and imaginary parts,

$$
\eta_{\alpha \beta}(\omega)=\eta_{\alpha \beta}^{\prime}(\omega)+i \eta_{\alpha \beta}^{\prime \prime}(\omega)
$$

Then the real part of the frequency dependent viscosity is given as

$$
\eta_{\alpha \beta}^{\prime}(\omega)=\frac{V}{k_{B} T} \int_{0}^{\infty} d t\left\langle\sigma_{\alpha \beta}(t) \sigma_{\alpha \beta}(0)\right\rangle \cos \omega t .
$$


Another important correlation function which is a direct measure of the building up of orientational order is angular velocity autocorrelation function. The angular velocity autocorrelation function of a system of ellipsoids is given as

$$
C_{\omega}(t)=\frac{\left\langle\boldsymbol{\omega}_{i}(t) \cdot \boldsymbol{\omega}_{i}(0)\right\rangle}{\left\langle\boldsymbol{\omega}_{i}(0) \cdot \boldsymbol{\omega}_{i}(0)\right\rangle},
$$

where $\boldsymbol{\omega}_{i}$ is the angular velocity of the $i$ th molecule. The relation between the rotational friction and the angular velocity autocorrelation function in the Laplace plane is given by the relation ${ }^{32,33}$

$$
C_{\omega}(z)=\frac{k_{B} T}{I\left[z+\zeta_{R}(z)\right]} .
$$

We now present the details of the simulation.

\section{SYSTEM AND SIMULATION DETAILS}

Molecular dynamics simulations have been carried out for a system of 576 Gay-Berne ${ }^{34,35}$ ellipsoids at temperature $T^{*}=1$ near $I-N$ transition in a microcanonical ensemble. The form of the modified intermolecular Gay-Berne potential used in the simulation is ${ }^{14-17,30}$

$$
\begin{aligned}
U= & 4 \epsilon\left(\hat{r}, \vec{u}_{i}, \vec{u}_{j}\right)\left[\left(\frac{\sigma_{s}}{r-\sigma\left(\hat{r}, \vec{u}_{i}, \vec{u}_{j}\right)+\sigma_{s}}\right)^{12}\right. \\
& \left.-\left(\frac{\sigma_{s}}{r-\sigma\left(\hat{r}, \vec{u}_{i}, \vec{u}_{j}\right)+\sigma_{s}}\right)^{6}\right],
\end{aligned}
$$

where $\hat{r}$ is the unit vector that passes through the center of mass of a pair of molecules; $\vec{u}_{i}$ and $\vec{u}_{j}$ unit vectors that pass through the major axis of a pair of ellipsoidal molecules; $\epsilon$ and $\sigma$ give the strength and range of interaction,

$\sigma\left(\hat{r}, \vec{u}_{i}, \vec{u}_{j}\right)=\sigma_{s}\left[1-\frac{\chi}{2}\left(\frac{\left(\vec{u}_{i} \cdot \hat{r}+\vec{u}_{j} \cdot \hat{r}\right)^{2}}{1+\chi\left(\vec{u}_{i} \cdot \vec{u}_{j}\right)}+\frac{\left(\vec{u}_{i} \cdot \hat{r}-\vec{u}_{j} \cdot \hat{r}\right)^{2}}{1-\chi\left(\vec{u}_{i} \cdot u_{j}\right)}\right)\right]$,

$\sigma_{s}$ is double of the minor axis $b$; $\kappa$ gives molecular elongation (aspect ratio), which is the ratio of end-to-end to sideto-side diameters; and $\kappa=\sigma_{e} / \sigma_{s}$. The aspect ratio of ellipsoids used in this simulation is 3. Here $\chi$ is defined as $\kappa$ as $\chi=\left(\kappa^{2}-1\right) /\left(\kappa^{2}+1\right)$,

$$
\begin{aligned}
\epsilon\left(\hat{r}, \vec{u}_{i}, \vec{u}_{j}\right)= & \epsilon_{0}\left[1-\chi^{2}\left(\vec{u}_{i} \cdot \vec{u}_{j}\right)\right]^{-1 / 2}\left[1-\frac{\chi^{\prime}}{2}\left(\frac{\left(\vec{u}_{i} \cdot \hat{r}+\vec{u}_{j} \cdot \hat{r}\right)^{2}}{1+\chi\left(\vec{u}_{i} \cdot \vec{u}_{j}\right)}\right.\right. \\
& \left.\left.+\frac{\left(\vec{u}_{i} \cdot \hat{r}-\vec{u}_{j} \cdot \hat{r}\right)^{2}}{1-\chi\left(\vec{u}_{i} \cdot u_{j}\right)}\right)\right]^{2}
\end{aligned}
$$

where $\epsilon_{0}$ is the energy parameter and $\chi^{\prime}=\left(\sqrt{\kappa^{\prime}}-1\right) /\left(\sqrt{\kappa^{\prime}}\right.$ $+1)\left(\kappa^{\prime}=\epsilon_{s} / \epsilon_{e}\right.$ gives the strength of interaction which is side-to-side to end-to-end well depths). The value of $\kappa^{\prime}$ used in the simulation is $5 .^{15,18,36}$ The scaling used for moment of inertia is $I^{*}=I / m \sigma_{0}^{2}$. The density is scaled in the simulation as $\rho^{*}=\rho \sigma_{0}^{3}$ and the temperature is scaled as $T^{*}=k_{b} T / \epsilon_{0}$. The equation of motion is integrated with reduced time $\left[t^{*}\right.$ $\left.=\left(m \sigma_{0}^{2} / \epsilon_{0}\right)^{1 / 2}\right]$ steps with $\Delta t=0.002 t^{*}$. The ellipsoid used in the simulation has minor axis $b=0.5$ and major axis $a$ $=1.5$ (in reduced units). The simulations are done at the state points near the pretransition region of phase diagram, shown

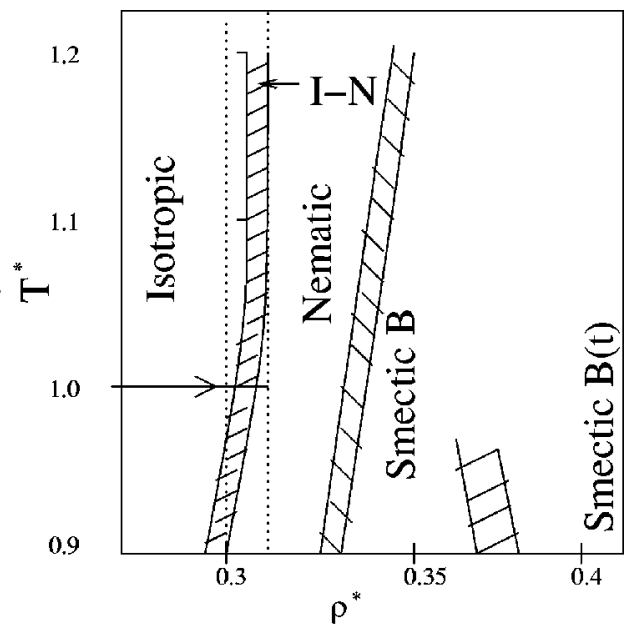

FIG. 1. The phase diagrams of the Gay-Berne ellipsoids are given here. The simulations are carried out at the temperature and densities indicated by the arrow.

in Fig. 1 The translational and rotational motions are solved using leap-frog algorithm. The order parameter changes dramatically in this system after density increases beyond 0.3 . This is in accord with previous simulations. ${ }^{17}$

The simulation starts from an equilibrated configuration of ellipsoids. Initial configuration of the ellipsoids is generated from a cubic lattice and then the simulation is run for two-hundred thousand steps to obtain the equilibrium configuration. During the equilibration steps the temperature is scaled so that the system is in equilibrium at this particular temperature. Four million production steps are used for calculation of viscosities.

In Fig. 1, we show the phase diagram of ellipsoids with aspect ratio 3 interacting with Gay-Berne potential. In the diagram we also show by arrow the densities studied at temperature $\left(T^{*}=1.0\right)$. Fig. 2 shows the variation of order parameter $S$ (Ref. 17) with density. We now present the results.

\section{RESULTS AND DISCUSSION}

The simulations have given rise to a host of important results, many of which were not anticipated earlier. Some of the results have been reported in a recent communication where we discuss the emergence of the power law in the orientational relaxation. In this work, we concentrate on the friction and the viscosity of the liquid as the $I-N$ transition is approached and on related several dynamical quantities.

\section{A. Shear viscoelasticity and stress-stress time correlation function}

It has been discussed elsewhere that the orientational correlation functions slow down dramatically as the $I-N$ transition is approached from low densities. ${ }^{7,8,17}$ The variation of shear viscosity near the $I-N$ transition is another subject of interest. It is of course known that viscosity does not exhibit any divergence near the $I-N$ transition. It is also 


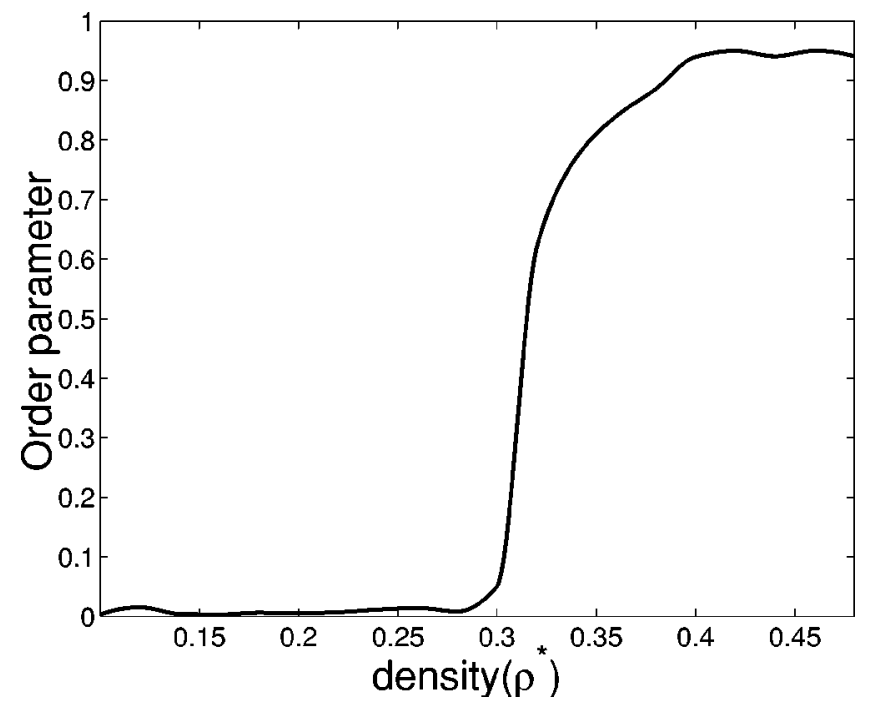

FIG. 2. The variating in the order parameter with density is shown in the figure.

known that the viscosity becomes anisotropic near the transition. However, a detailed study of the emergence of the anisotropy and the variation of viscosity seems to be absent in the literature.

In Fig. 3 we show the variation of shear viscosity with density. There are several points of interest about this figure. First, the increase in viscosity with density becomes sharper as the $I-N$ transition is approached. However, this growth is rather mild. Second point to note is that beyond the transition point, the viscosity becomes anisotropic. The emergence of anisotropy can be better understood by studying the probability distribution of the mean square stress. In Fig. 4 we show the distribution at three densities. Note that the distribution is fully isotropic at low densities but becomes anisotropic as the transition is approached. This anisotropy is important in determining the viscous response of the liquid. The anisotropy in the values of different components of viscosity

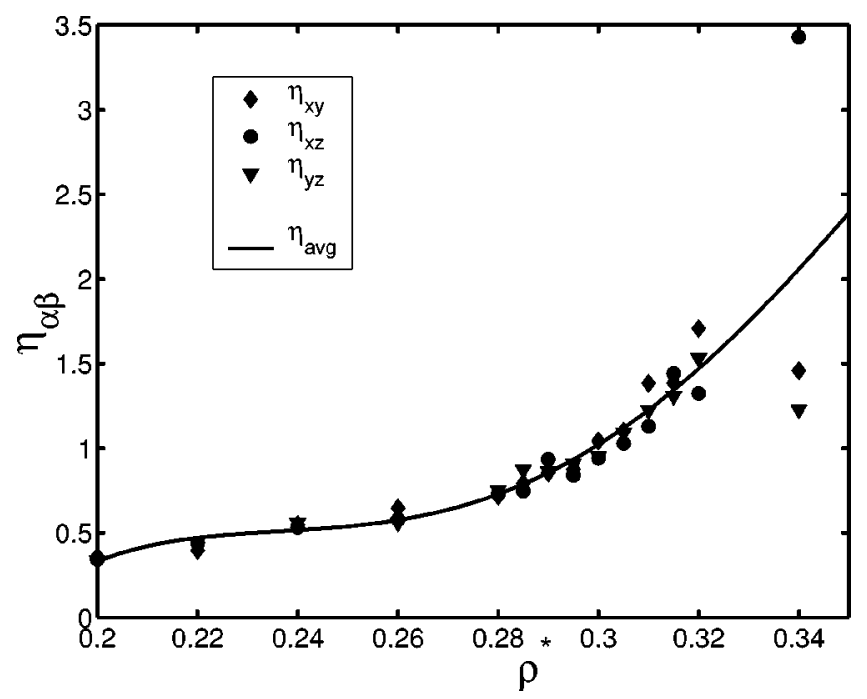

FIG. 3. The viscosity of a system of Gay-Berne ellipsoids is plotted against density in the isotropic phase. When the $I-N$ transition is approached, various components of viscosity increase and they become anisotropic.

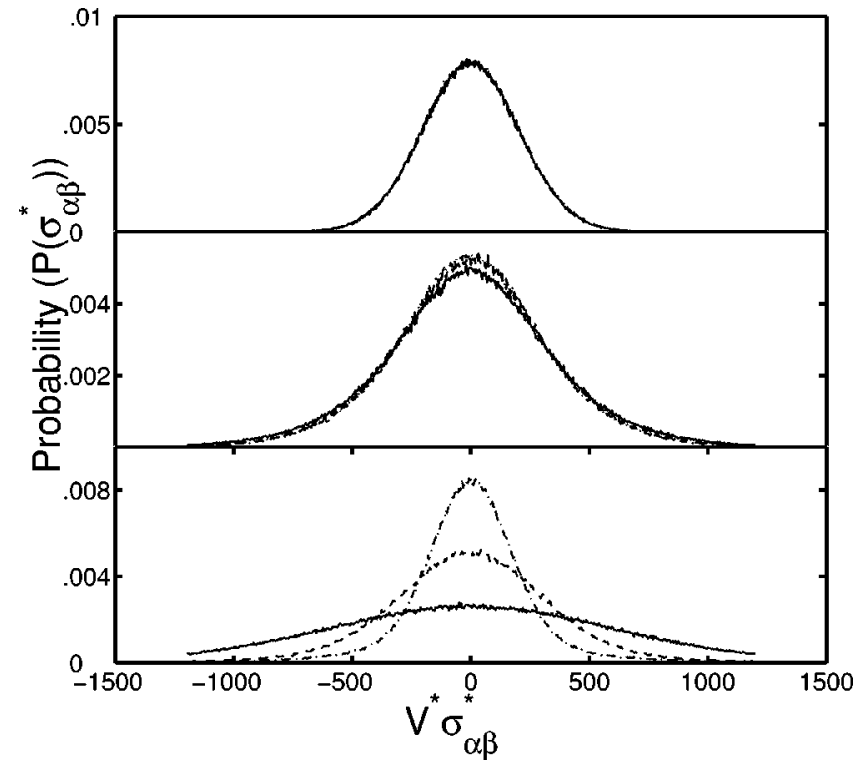

FIG. 4. The distribution of three different components of stress $\left[\sigma_{x y}\right.$ (dashed line), $\sigma_{x z}$ (continuous line), and $\sigma_{y z}$ (dot-dashed line)] is shown here. The top figure is at density $\rho^{*}=0.20$ (isotropic phase); the middle figure is at density $\rho^{*}=0.315$ (near $I-N$ transition); and the bottom figure is at density $\rho^{*}=0.34$ (nematic phase).

near $I-N$ transition is clearly shown in Table I. We note that the stress tensor also becomes anisotropic in the deeply supercooled liquid. ${ }^{37}$ Although this analogy is rather nice, the origin of the two is obviously quite different.

In Fig. 5 we show the decay of the stress-stress time correlation function. Note the negative region and the slow long time decay. We shall return to this point below.

We now discuss the viscoelasticity of the liquid which shows several interesting features. In Fig. 6 we show the frequency dependence of the viscosity. There are three unusual features of this figures:

(a) The fall of viscosity with frequency is nonlinear and unusually weak at low frequencies. It is clear that the frequency dependence obeys a power law dependence here with a small exponent. Such weak frequency dependence of viscosity has been observed earlier by Tang et $a .^{28}$ and is related to the long time power law decay of the stress time correlation function.

(b) There is a shallow minimum at somewhat higher

TABLE I. Values of viscosities starting from very low densities to densities near $I-N$ transition.

\begin{tabular}{cccc}
\hline \hline Density & $\eta_{x y}^{*}$ & $\eta_{x z}^{*}$ & $\eta_{x z}^{*}$ \\
\hline 0.05 & 0.07 & 0.07 & 0.07 \\
0.10 & 0.11 & 0.11 & 0.11 \\
0.15 & 0.20 & 0.20 & 0.20 \\
0.20 & 0.38 & 0.38 & 0.37 \\
0.25 & 0.64 & 0.64 & 0.65 \\
0.3 & 1.16 & 1.05 & 1.07 \\
0.31 & 1.53 & 1.31 & 1.32 \\
0.32 & 1.75 & 1.42 & 1.72 \\
0.34 & 1.59 & 3.65 & 1.26 \\
\hline \hline
\end{tabular}




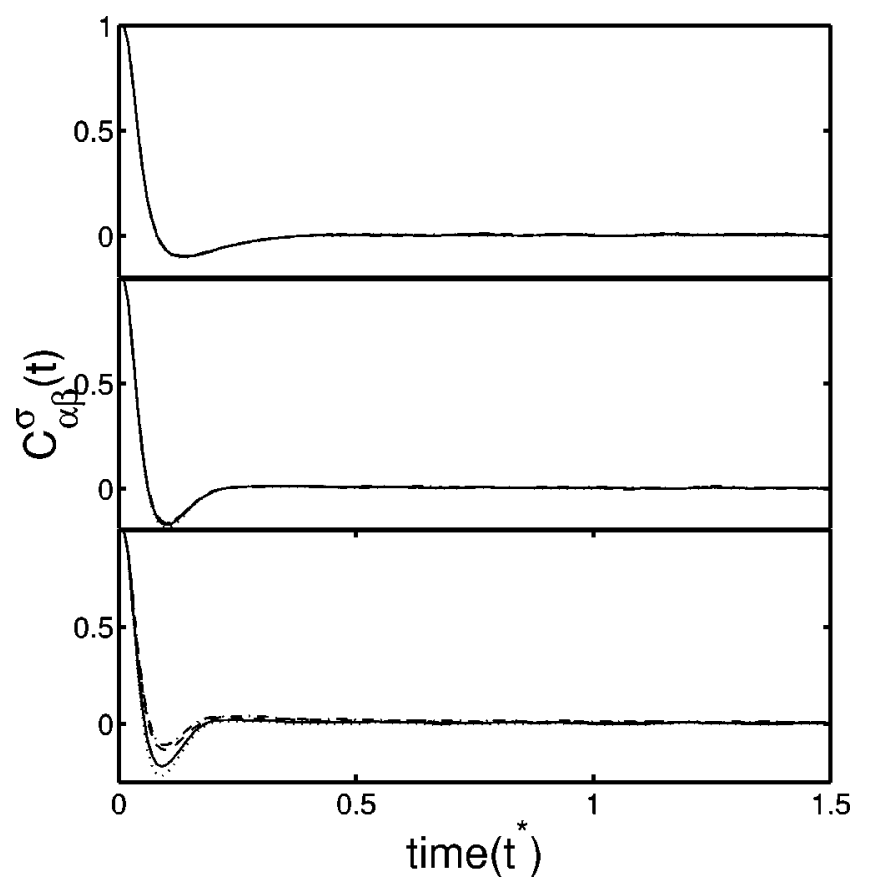

FIG. 5. Components of the normalized stress-stress correlation function (the dashed line gives the $x y$ component, the dashed-dotted line gives the $x z$ component, the dotted line gives the $y z$ component, and the continuous line gives the average) is plotted here at three densities. The top figure is at density $\rho^{*}=0.20$ (isotropic phase); the middle figure is at density $\rho^{*}$ $=0.315$ (near $I-N$ transition); and the bottom figure is at density $\rho^{*}$ $=0.34$ (nematic phase).

frequency, near the reduced value of frequency equal to unity.

(c) There is a pronounced peak near a value of reduced frequency which is $\approx 20$. In real units, the peak is around frequency $10^{13} \mathrm{~s}^{-1}$. We now turn to the possible interpretation of this interesting behavior.

The explanation, of course, lies in the time dependence of the stress-stress TCF shown in Fig. 5. Note the slow decay in the long time. In an interesting paper, Zwanzig pointed out

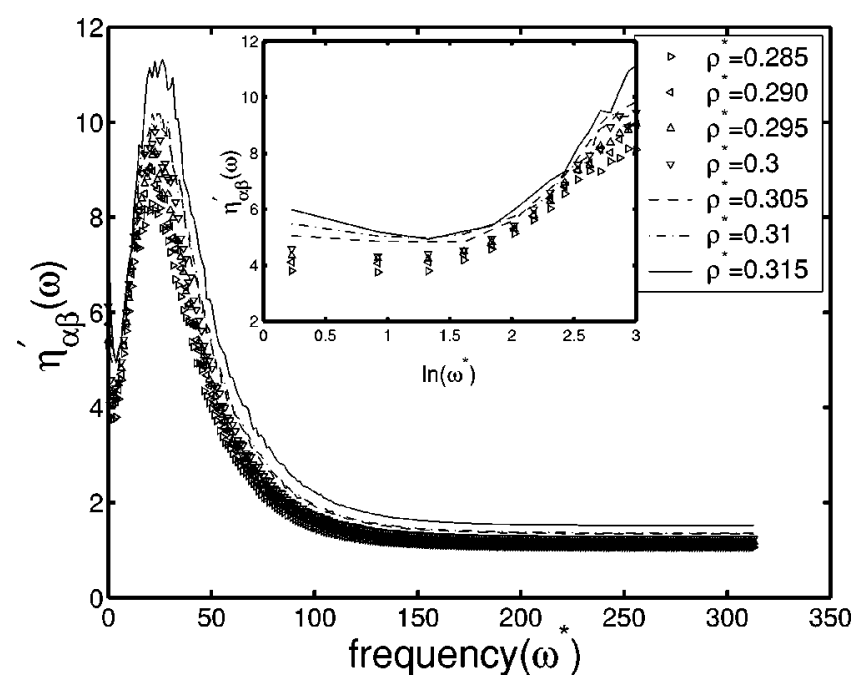

FIG. 6. The frequency dependent viscosity is plotted against the frequency at different densities near $I-N$ transition. The inset gives the plot $\eta_{\alpha \beta}(\omega)$ vs $\ln (\omega)$. This shows the low frequency characteristics of $\eta_{\alpha \beta}(\omega)$.

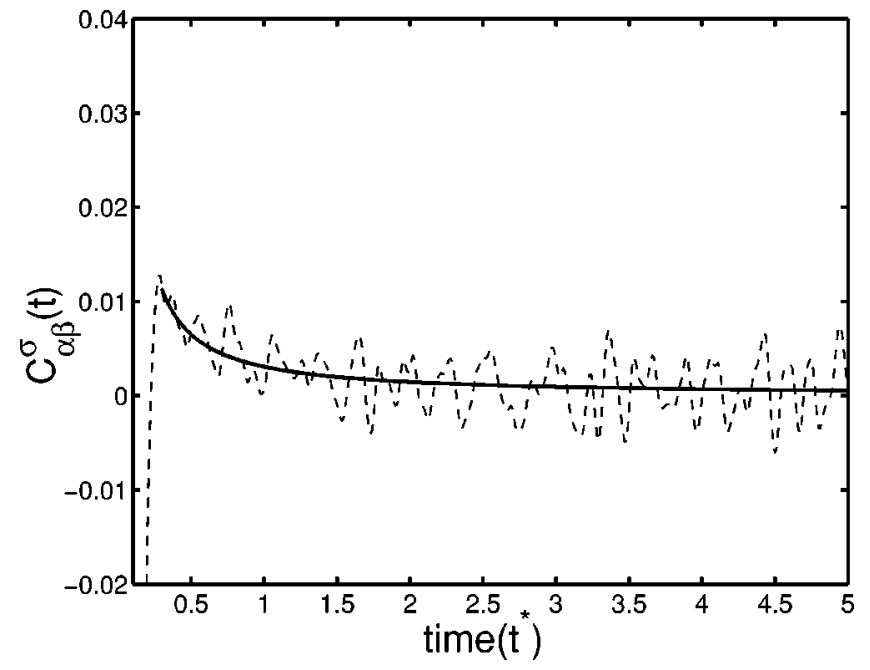

FIG. 7. The tail of the stress-stress correlation function is fitted to the function $y=0.0031 t^{-1.1}$ (the continuous line gives the fit and the dashed line gives the stress-stress correlation function) at density $\rho^{*}=0.315$. The exponent $\alpha$ obtained from the fit is 1.1 .

the correlation between the slow long time decay of the stress-stress TCF and a nonanalytic frequency dependence of the viscosity. ${ }^{38}$ That is, if the long time tail decays as $t^{-3 / 2}$, then the frequency dependence is given by

$$
\eta^{\prime}(\omega)=\eta(0)-A \omega^{1 / 2}+O(\omega) .
$$

When we fit the long time part of the stress TCF to a form $t^{-\alpha}$, we find a value of $\alpha \sim 1.1$ for the exponent $\alpha$. The fit is shown in Fig. 7. The value of the exponent does provide an explanation for the weak frequency dependence at small frequencies of $\eta_{\alpha \beta}^{\prime}(\omega)$.

The minimum in $\eta_{\alpha \beta}(\omega)$ at small frequencies seems to originate from the negative region in the stress-stress TCF. Note that the negative region of $C_{\alpha \beta}^{\sigma}(t)$ becomes more pronounced as the $I-N$ transition is approached, so does the peak in $\eta_{\alpha \beta}(\omega)$. The maximum is due to damped oscillations in the stress time correlation function. Note the close similarity between the frequency dependence of viscosity and that of dynamics structure factor, ${ }^{21}$ signifying the general validity of the mode coupling theory expression relating STCF to the dynamic structure factor. The sharp peak in $\eta(\omega)$ occurs at $\omega^{*} \approx 20$ which is a rather large frequency. In real terms, the value of the frequency at the peak is about $10^{13} \mathrm{~s}^{-1}$. The sharp increase in the peak value as the $I-N$ transition is approached from low density indicates the formation of a nearest neighbor cage giving rise to high frequency, rattling-type motion. Such a cage formation is also a prerequisite for glass transition. This cage can also be regarded as a manifestation of translation-rotation coupling because the cage is essentially due to local orientational ordering.

\section{B. Angular velocity correlation}

In Fig. 8 we show the time dependence of the angular velocity TCF. Note the slow decay in the long time, follow- 


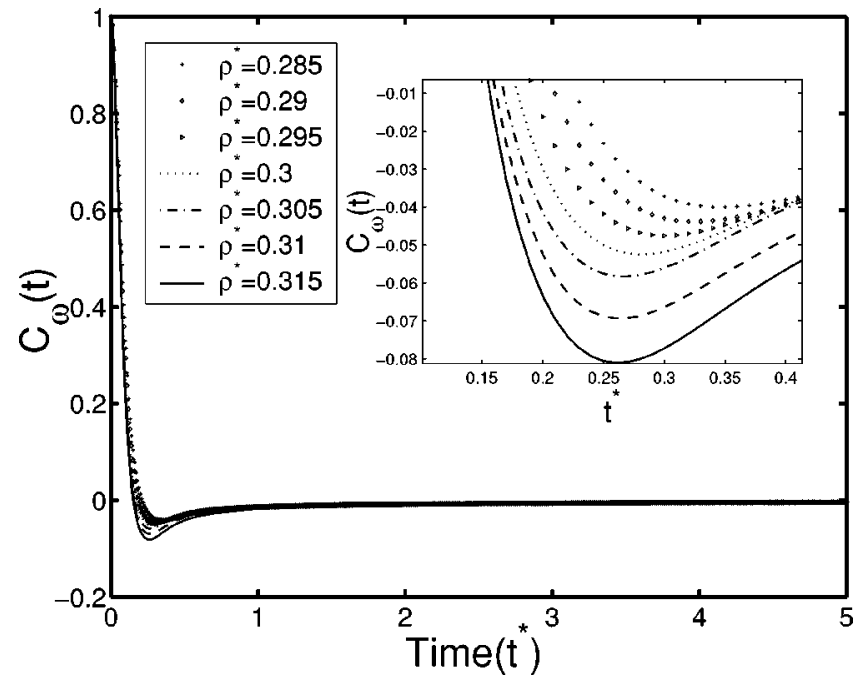

FIG. 8. The angular velocity autocorrelation function is plotted at different densities. As the density of the system increases the depth of backscattering region of the angular velocity autocorrelation function becomes deep. This is clearly shown in the inset where the negative region of the angular velocity autocorrelation function is highlighted.

ing the negative dip at short times. The rise of the velocity TCF after the minimum has been fitted to a function of the following form:

$$
\langle\omega(0) \omega(t)\rangle \approx-a_{1} \exp \left(-a_{2} t\right)-a_{3} t^{-a_{4}} .
$$

The fit has been shown in Fig. 9. The power law exponent is about 1.2. Hydrodynamic prediction of long time power law exponent is much larger, equal to $5 / 2 .{ }^{24-27}$ This point deserves further study. It is possible that the long time decay observed in simulation is not the true hydrodynamic long time decay-instead an intermediate time decay one often finds in the mode coupling theory which arises from the coupling with the density mode.

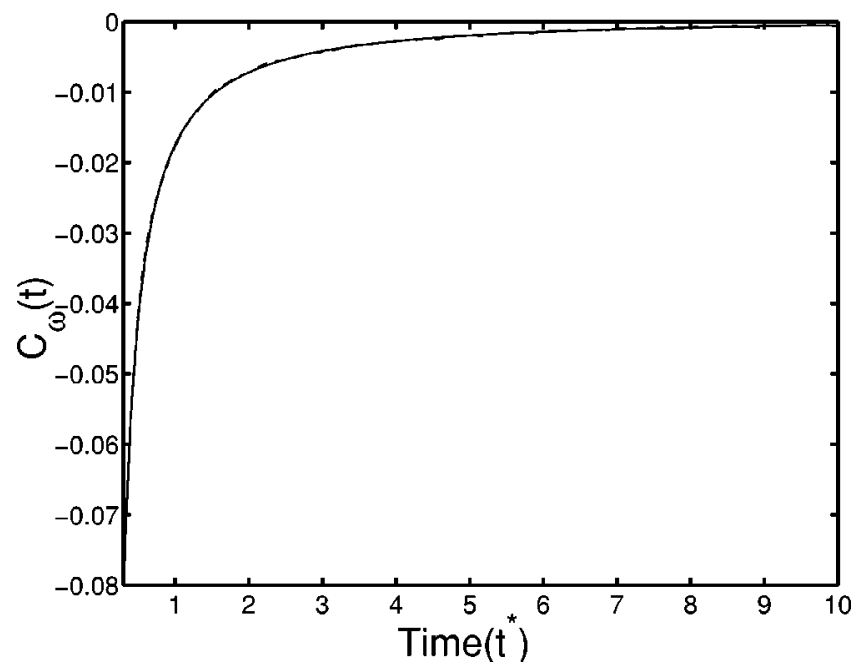

FIG. 9. The tail of the angular velocity autocorrelation function fitted to a power law (the continuous line gives the fit and the dashed line gives the stress-stress correlation function). The exponent $\alpha$ obtained from the fit is 1.2 at density $\rho^{*}=0.315$.

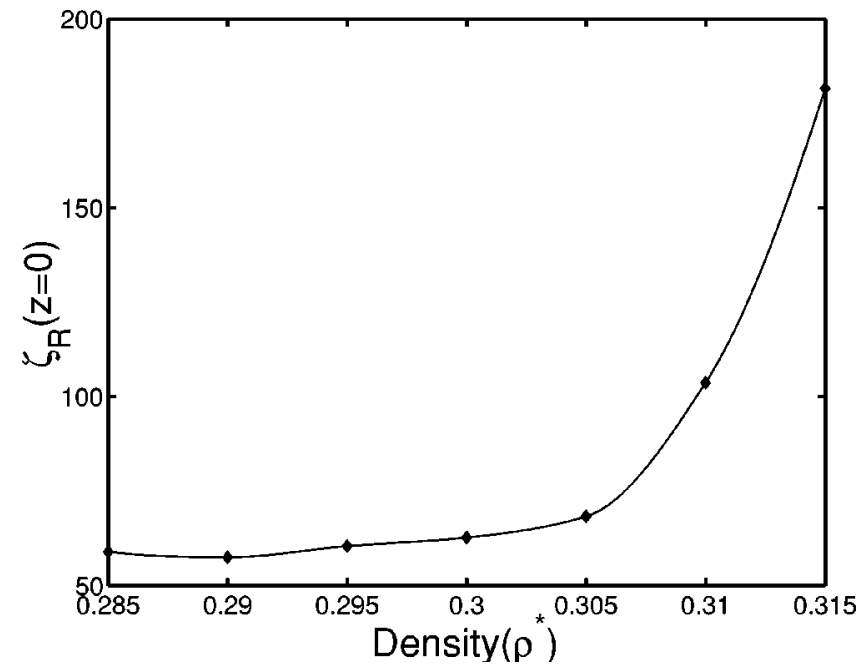

FIG. 10. The zero-frequency friction is plotted against density. The friction rapidly increases as $I-N$ transition is approached along the density axis.

The last point can be further substantiated if one uses the MCT form of the friction ${ }^{32,33,39}$ in Eq. (10). The expression for the single particle rotational friction can be written as

$$
\begin{aligned}
\zeta_{R}(z)= & \zeta_{\text {bare }}+\frac{\rho}{2(2 \pi)^{4}} \int_{0}^{\infty} d t e^{-z t} \int_{0}^{\infty} d k k^{2} \\
& \times \sum_{l_{1} l_{2} m} c_{l_{1} l_{2} m}^{2}(k) F_{l_{2} m}^{s}(k, t),
\end{aligned}
$$

where $c_{l_{1} l_{2} m}(k)$ is the $l_{1}, l_{2}, m$ th coefficient of the twoparticle direct correlation function and

$$
F_{l m}^{s}(k, t)=\left\langle e^{-i \mathbf{k} \cdot \mathbf{r}} Y_{l m}\left[\boldsymbol{\Omega}_{i}(0)\right] Y_{l m}\left[\boldsymbol{\Omega}_{i}(t)\right]\right\rangle
$$

is the single particle orientational correlation function. One finds nearly perfect agreement at long times, substantiating the logic presented here. However, a more complete calculation is required to fully reproduce the angular velocity correlation curve.

The initial decay of the angular velocity correlation function is fast and inertial. This may be due to rattling motion of the ellipsoids within its orientational cage (cone) formed by the nearest neighbors. Such a motion can explain the peak in the viscoelasticity. Of course the same rattling motion will be present in the translational motion.

\section{Rotational friction on the ellipsoids}

We have discussed earlier that the single particle orientational correlation function exhibits power law decay behavior at intermediate times. In Fig. 10 we show the density variation of the zero frequency friction at the temperature $T^{*}=1.0$. Note the rapid rise in the friction as the transition density is approached.

Mode coupling theory predicts that due to the emergence of the long range orientational density correlation, the rotational friction should show a strong frequency dependence. Here the frequency is the Laplace frequency. In Fig. 11 we show the frequency dependence of rotational friction at sev- 


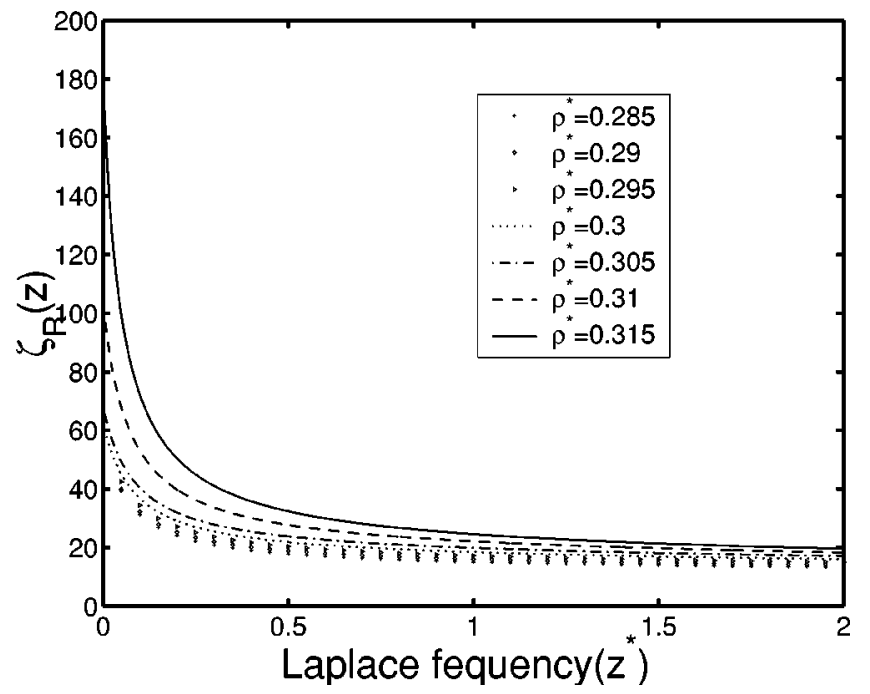

FIG. 11. The frequency dependent friction is plotted at different densities.

eral densities. Note the increasingly faster rise of the friction at low frequencies as the $I-N$ transition is approached.

\section{Comparison between viscosity and rotational friction}

While the friction shows a sharp increase near the $I-N$ transition, the same is absent for viscosity. In Fig. 12, we have plotted the ratio of $\zeta_{R} / \eta$. The figure shows a hydrodynamiclike, density independent (or weakly dependent) behavior at low densities which gives away to a strong variation as the $I-N$ transition point is approached. This is the manifestation of the strong growth of friction near the $I-N$ transition where viscosity shows only weak dependence on density. Thus, the Debye-Stokes relation between rotational friction and viscosity breaks down near the $I-N$ transition.

\section{CONCLUDING REMARKS}

Let us first summarize the main results of this paper. We have carried out molecular dynamics simulations of a system

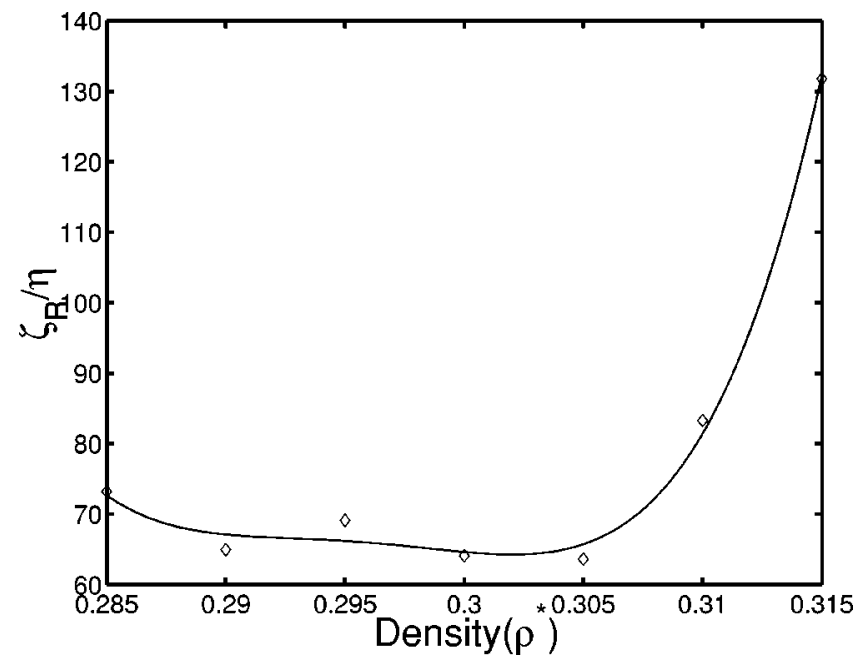

FIG. 12. The ratio of zero-frequency friction to the average viscosity is plotted against the density near $I-N$ transition. of Gay-Berne ellipsoids of aspect ratio 3 near its isotropicnematic phase transition. We have calculated the viscoelastic properties of the system. While the rotational friction shows a sharp rise in its value as the isotropic-nematic phase transition is approached, the viscosity shows only a mild increase. The frequency dependent viscosity shows interesting anomalies-a weak power-law dependence at low frequencies and a sharp peak at the intermediate frequency; the two are separated by a shallow minimum. While the observed behavior for Gay-Berne fluids near the $I-N$ transition is yet to be understood from a quantitative theory the anomalies in the viscoelastic properties can be qualitatively understood from the stress-stress time correlation function which shows a negative dip at short times and a power law decay at long times. The stress becomes anisotropic near the transition region which is expected.

One of the motivations of the present study was the recent work by Cang et al. who showed that Kerr response near the $I-N$ transition is surprisingly close to that in supercooled liquids. ${ }^{4,5}$ We find that although the orientational correlation function indeed shows power law decay, viscosity, and viscoelasticity do not show similar behavior. This is because the origin of the slow decay is completely different in the two cases. In supercooled liquid, the slow decay is believed to arise from a nonlinear feedback mechanism between the density and the stress relaxation, where the slow density relaxation occurs at nearest neighbor distance (near the first peak of the static structure factor). In the present system, the slowness arises from the long range orientational correlation. Nevertheless, the frequency dependence of viscosity already shows behavior similar to the ones observed in dense liquids.

There is one more similarity between the present system and supercooled liquids. The frequency dependent viscosity shows a sharp increase in its peak value at intermediate frequency as the $I-N$ transition is approached from low density. This clearly indicates formation of a nearest neighbor cage giving rise to high frequency rattling-type motion. Such a cage formation is also a prerequisite for glass transition.

This strong similarity between the two phenomena is really intriguing. The main reason appears to be the rapid growth in the value of the friction and the memory function of the stress time correlation function. Mode coupling theory shows that the rotational friction grows approximately as $1 / \sqrt{z}$ at low frequencies $(z)$. This gives rise to the power law decay. A similar rapid growth in the friction also takes place in the supercooled liquid.

While the theory of Tang et al. ${ }^{28}$ provides a framework to understand many aspects of the relaxation behavior reported here, it will be worthwhile to develop a mode coupling theory to understand the effect of orientational relaxation on viscoelasticity. Work in this direction is under progress.

\section{ACKNOWLEDGMENTS}

It is a pleasure to thank Professor M. Fayer (Stanford), Dr. Sarika Maitra Bhattacharyya (IISc), and Arnab Mukherjee for many discussions. This work was supported in parts by grants from DST and CSIR, India. 
${ }^{1}$ L. Onsager, Ann. N.Y. Acad. Sci. 51, 621 (1949).

${ }^{2}$ R. Zwanzig, J. Chem. Phys. 39, 1714 (1963).

${ }^{3}$ J. J. Stankus, R. Torre, and M. D. Fayer, J. Chem. Phys. 97, 9478 (1993).

${ }^{4}$ H. Cang, J. Li, and M. D. Fayer, Chem. Phys. Lett. 366, 82 (2002).

${ }^{5}$ H. Cang, J. Li, V. N. Novikov, and M. D. Fayer, J. Chem. Phys. 118, 9303 (2003).

${ }^{6}$ A. Sengupta and M. D. Fayer, J. Chem. Phys. 102, 4193 (1995).

${ }^{7}$ S. D. Gottke et al., J. Chem. Phys. 116, 360 (2002).

${ }^{8}$ S. D. Gottke, H. Cang, B. Bagchi, and M. D. Fayer, J. Chem. Phys. 116, 6339 (2002)

${ }^{9}$ A. Perera, G. N. Patey, and J. J. Weis, J. Chem. Phys. 89, 6941 (1988).

${ }^{10}$ M. Baus, J. L. Colot, X. G. Wu, and H. Xu, Phys. Rev. Lett. 59, 2184 (1987).

${ }^{11}$ J. L. Colot, X. G. Wu, H. Xu, and M. Baus, Phys. Rev. A 38, 2022 (1988).

${ }^{12}$ M. P. Allen and D. Frenkel, Phys. Rev. Lett. 58, 1748 (1987).

${ }^{13}$ M. P. Allen and M. A. Warren, Phys. Rev. Lett. 78, 1291 (1997)

${ }^{14}$ S. Ravichandran, A. Perera, M. Moreau, and B. Bagchi, J. Chem. Phys. 107, 8469 (1997).

${ }^{15}$ S. Ravichandran, A. Perera, M. Moreau, and B. Bagchi, J. Chem. Phys. 109, 7349 (1998).

${ }^{16}$ R. Vasanthi, S. Ravichandran, and B. Bagchi, J. Chem. Phys. 115, 10022 (2001).

${ }^{17}$ P. J. Prasanth and B. Bagchi, J. Chem. Phys. 120, 11256 (2004).

${ }^{18}$ A. M. Smondyrev, G. B. Loriot, and R. A. Pelcovits, Phys. Rev. Lett. 75, 2340 (1995).

${ }^{19}$ M. Miesowicz, Nature (London) 158, 27 (1946).
${ }^{20}$ U. Balucani and M. Zoppi, Dynamics of the Liquid State (Clarendon, Oxford, 1994).

${ }^{21}$ J. P. Hansen and I. R. McDonald, Theory of Simple Liquids (Academic, London, 1986).

${ }^{22}$ T. Geszti, J. Phys. C 16, 5805 (1983).

${ }^{23}$ B. Bagchi and S. Bhattacharyya, Adv. Chem. Phys. 116, 67 (2001).

${ }^{24}$ B. J. Berne, J. Chem. Phys. 56, 2164 (1972).

${ }^{25}$ J. A. Montgomery and B. J. Berne, J. Chem. Phys. 66, 2161 (1977).

${ }^{26}$ B. Cichocki and B. U. Felderhof, Physica A 213, 465 (1995).

${ }^{27}$ C. P. Lowe, D. Frenkel, and A. J. Maters, J. Chem. Phys. 103, 1582 (1995).

${ }^{28}$ S. Tang, G. T. Evans, C. P. Mason, and M. P. Allen, J. Chem. Phys. 102, 3794 (1995).

${ }^{29}$ M. P. Allen and D. J. Tildesley, Computer Simulation of Liquids (Clarendon, Oxford, 1987).

${ }^{30}$ J. Crain and A. V. Komolkin, Adv. Chem. Phys. 109, 39 (1999).

${ }^{31}$ W. G. Hoover et al., Phys. Rev. A 22, 1690 (1980).

${ }^{32}$ B. Bagchi and A. Chandra, Adv. Chem. Phys. 80, 1 (1991).

${ }^{33}$ B. J. Berne and R. Pecora, Dynamic Light Scattering: With Applications to Chemistry, Biology and Physics (Wiley, New York, 1976).

${ }^{34}$ B. J. Berne and P. Pechukas, J. Chem. Phys. 56, 4213 (1972).

${ }^{35}$ J. G. Gay and B. J. Berne, J. Chem. Phys. 74, 3316 (1981).

${ }^{36}$ E. DeMiguel, L. F. Rull, and K. E. Gubbins, Phys. Rev. A 45, 3813 (1992).

${ }^{37}$ S. Bhattacharyya and B. Bagchi, Phys. Rev. Lett. 89, 025504 (2002).

${ }^{38}$ R. Zwanzig, Proc. Natl. Acad. Sci. U.S.A. 78, 3296 (1981).

${ }^{39}$ B. Bagchi, J. Mol. Liq. 77, 117 (1998). 